\title{
Carbon Footprint and Order Quantity in Logistics
}

\author{
Zhiyong Tian, Lingyu Huo, Guicheng Shen \\ School of Information, Beijing Wuzi University (China) \\ tianbox@126.com;sboerp@163.com; guichengshen@126.com
}

\begin{abstract}
:
Purpose: Even without economic factors and government regulations, the pressure and motivation of corporation to reduce emissions are still increasing. This is because the key factors for corporation to reduce emissions have been corporate social responsibility and increasing awareness of low carbon among consumers and society not economic trade-off and stringent government regulations. So, the purpose of this paper is to provide quantity methods for the logistics organizations with wish of voluntary reduction and social responsibility to reduce emissions through operations adjustment.
\end{abstract}

Design/methodology/approach: Being difference from the traditional research that takes economic value as objective and carbon footprint as constraint or another source of economic cost, this paper takes carbon footprint as objective directly, order quantity as decision variable. By referring to the traditional economic order quantity model, the paper proposes logistics carbon footprint model which takes transport and inventory into account. Then it solves the model by calculating the values of order quantity, carbon footprint and revenue using the method of optimization.

Findings: By solving and comparing the two models, economic order quantity model and carbon footprint model, it gets some results, such as carbon optimization order quantity, the effects of order quantity deviating from economic or carbon order quantity on economic or carbon footprint values, which can give some meaningful insights for corporation to look for reduction opportunities by operations adjustment. 
Originality/value: The study takes carbon footprint as objective directly and creates the corresponding quantity model. By comparing with the traditional economic order quantity model, it can provide quantity methods and some meaningful insights for the logistics organizations to reduce emissions.

Keywords: inventory, transport, carbon footprint, order quantity

\section{Introduction}

Logistics plays a special role in low carbon economy. On the one hand, logistics itself is an important source of large volumes of carbon emissions. On the other hand, low carbon economy should be supported by efficient logistics. So, low carbon logistics has become a new hot research area. Recently, there has emerged a stream of literatures related to this area. As one of the earlier research, Benjaafar, Yanzhi and Daskin (2013) modified traditional logistics model by taking carbon as constraints. The research is for the policies of carbon cap, carbon tax, carbon cap and trade and carbon offset. Hua, Cheng and Wang (2011) extended Benjaafar's (2013) research. By converting the carbon constraints equation, they developed unconstrained optimization model that is for carbon trade and carbon cap. Arslan and Turkay (2010) applied this method to the context of sustainable development. They revisited economic order quantity (EOQ) with sustainability consideration in the cases of direct accounting, carbon tax, direct cap, cap and trade and carbon offsets. Battini, Persona and Sgarbossa (2013) developed sustainable EOQ model by incorporating environmental criteria into EOQ. They integrated environment factors into the objective function by assuming the cost coefficients of these factors. Chen, Benjaafar and Elomri (2012) did an interesting research by developing a new EOQ optimization model with carbon constraints. By a series of complicated and sophisticated transformations, they got some meaningful and important insights, which supported Benjaafar's (2013) numerical observations. Bouchery et al. (2012) tried to modify classical EOQ model as the sustainable order quantity model with multi-objective. The research is for carbon regulation. The literatures above developed inventory model with taking into account carbon footprint, environmental or social criteria. It is the prevalent method in the present researches on low carbon logistics. There are still some other researches beyond inventory management area. The representative literatures are as follows. Hoen et al. (2011) researched the effect of different types of emission regulation on transport mode selection. The regulations are carbon cost, carbon tax and carbon cap. Cachon (2013) researched the density, size and location of retail store with considering the cost of carbon. Based on the traditional solution of this classical problem, such as k-median and traveling salesman problem, he developed standard optimization model including carbon cost by assuming the value of carbon cost coefficient. Carlsson and Jia (2012) researched a continuous facility location problem with carbon emission. The optimization objective is to minimize the weighted sum of three costs, 
one of which is carbon cost. The question is similar to Cachon's (2013), so is the approach for treating carbon emission.

Some international organizations, such as IPCC and CARBON TRUST, have released a lot of documents about how to accurately measure and calculate carbon footprint. But these are out of this paper. From the perspectives of the literatures presented above, there are two main types of approaches to treat carbon footprint according to two policies. One, for carbon trade, is to set carbon cost coefficient and incorporate it into the objective function, namely the carbon footprint is thought as an additional source of economic cost. The other, for carbon cap, is to regard carbon as the constraints of traditional model. Carbon trade has been implemented in some countries, e.g., the European Union has already implemented the carbon emission trading scheme (EU ETS) for the energy-intensive industries, which currently account for almost $50 \%$ of Europe's carbon emissions. But carbon trade is insignificant for emission reduction. Hoen et al. (2011) believe that carbon emission cost accounts for a very small part of the total cost in supply chains. So they conclude that adding emission costs leads rarely to a change in the selected transport mode. Cachon (2013) gets the same conclusion that substantial reduction only occur if the carbon price is extremely high (e.g. greater than $\$ 1,000$ per metric tonne). So a price on carbon is an ineffective mechanism for reducing emissions. On the other hand, carbon regulations are not substantially implemented in many countries. So the two settings that most literatures supposed are very different from the current reality. Fortunately, with the evolution of society, people's awareness of environmental protection is gradually improving. More and more consumers are becoming aware of sustainable development issues (Wakeland, Sears \& Venkat, 2009; Blengini \& Shields, 2010). Firms thus seek to get competitive advantage by selling greener products. Many progressive companies, such as Wal-Mart, Tesco, HP and Patagonia, have capitalized on the opportunities of green supply chain management. This trend is reflected in a 2008 survey of 40 chief executive officers from many of the largest third-party logistics industries worldwide (Lieb \& Lieb, 2010). The top three reasons to establish sustainability programs are 'The corporate desire to do the right thing', 'The pressure from customers' and 'The corporate desire to enhance company image'. Another recent survey of 582 European companies highlights that the regulation is no longer considered as the most important reason to establish sustainability programs (BearingPoint, 2010-2011). Unfortunately, by now there are few direct researches for these firms which have the motivation to reduce emissions voluntarily. So, instead of focusing on the cost of carbon, the paper takes carbon footprint as the optimization target with taking cost optimization as reference. It tries to provide quantity methods for the corporations with wish of voluntary reduction and social responsibility. The rest of this paper is organized as follows: In Section 2 we formulate the carbon footprint order quantity model and the reference model of EOQ. In Section 3 we solve the model and obtain some meaningful results by analyzing the decision viable and objective value under each case. In Section 4 we provide some numerical examples to illustrate and verify the insights obtained in Section 3. In Section 5, we conclude the paper and suggest some topics for further research. 


\section{The Model}

The activities of logistics can lead to either economic costs or carbon footprints. Carbon footprints are equal to carbon emission factor (EF) multiplied by action data (AD). EF is greenhouse gases (GHG) emissions per unit activity. It is measured by $\mathrm{CO}_{2}$ equivalent. $\mathrm{AD}$ is the quantity of activities releasing GHG. For economic costs, it is easier to get the accurate data of activities generally, which can be used in calculating carbon footprints. For carbon footprints, the coefficient is more difficult to obtain. Though there are some public available data, they may not be a proper match for AD. Fortunately, our purpose is not to obtain public, authoritative and accurate data of carbon, but to support making the decision of reduction. As for lack of accurate detailed data, Daganzo (2010) believes that detailed data may be harmful to making rational decision in the analysis of logistics. Caro, Charles, Tan and Rob (2011) thinks that focusing on the life-cycle assessment and carbon footprint literature avoiding double-counting is misguided, for over-allocated emission is benefit for making efficient measure to reduce emissions. Then we believe that it can achieve the research purpose of this paper only by estimating the data about carbon footprint roughly. So, the focus of this paper is not on acquiring accurate authority data.

Our study is limited to transport and inventory, which account for a large proportion of logistics either in cost or in carbon. There are two types of carbon footprint. One is direct carbon footprint, also called variable carbon footprint, due to the activities related to output directly. The other is indirect carbon footprint, also called fixed carbon footprint, due to the supporting activities being no related to output directly. The relationship between the two types of carbon footprint is similar as that of the variable and fixed costs. The notations employed in the model are as follows:

- Atf fixed activity of transport

- $F_{t f}$ EF of fixed activity of transport

- $\quad C_{t f}$ cost per unit fixed activity of transport

- $A_{t v}$ variable activity of transport

- $F_{t v}$ EF of variable activity of transport

- $C_{t v}$ cost per unit variable activity of transport

- $A_{h f}$ fixed activity of inventory

- $\quad F_{h f}$ EF of fixed activity of inventory

- $C_{h f}$ cost per unit fixed activity of inventory 
- $A_{h v}$ variable activity of inventory

- $F_{h v}$ EF of variable activity of inventory

- $\quad C_{h v}$ cost per unit variable activity of inventory

- $Q$ order quantity (decision variable)

- $\quad D$ demand per unit time (demand speed)

We adopt the conventional hypothesis of classical inventory theory, namely deterministic demand. So, order cycle is $t=Q / D$. For simplicity without loss of generality, we suppose linear relationship between variable activity and carbon or cost, which is commonly used in the literatures.

\subsection{Transport}

The carbon footprint per unit time is:

$T F_{t}(Q)=\left(F_{t f} \cdot A_{t f}+F_{t v} \cdot A_{t v} \cdot Q\right) / \frac{Q}{D}=F_{t f} A_{t f} \frac{D}{Q}+F_{t v} A_{t v} D$

The transport cost per unit time is:

$T C_{t}(Q)=\left(C_{t f} \cdot A_{t f}+C_{t v} \cdot A_{t v} \cdot Q\right) / \frac{Q}{D}=C_{t f} A_{t f} \frac{D}{Q}+C_{t v} A_{t v} D$

\subsection{Inventory}

The carbon footprint per unit time is:

$T F_{h}(Q)=\left(F_{h f} \cdot A_{h f}+F_{h v} \cdot A_{h v} \cdot \frac{Q}{2}\right.$

The inventory cost per unit time is:

$T C_{h}(Q)=\left(C_{h f} \cdot A_{h f}+C_{h v} \cdot A_{h v} \cdot \frac{Q}{2}\right.$ 
The total carbon footprint per unit time is:

$$
T F(Q)=T F_{t}(\mathrm{Q})+T F_{h}(\mathrm{Q})=\mathrm{F}_{t f} \mathrm{~A}_{t f} \frac{\mathrm{D}}{\mathrm{Q}}+\mathrm{F}_{t v} \mathrm{~A}_{t v} \mathrm{D}+\mathrm{F}_{h f} \mathrm{~A}_{h f}+\mathrm{F}_{h v} \mathrm{~A}_{h v} \frac{\mathrm{Q}}{2}
$$

The total cost per unit time is:

$$
T C(\mathrm{Q})=T C_{t}(\mathrm{Q})+T C_{h}(\mathrm{Q})=\mathrm{C}_{t f} \mathrm{~A}_{t f} \frac{\mathrm{D}}{\mathrm{Q}}+\mathrm{C}_{t v} \mathrm{~A}_{t v} \mathrm{D}+\mathrm{C}_{h f} \mathrm{~A}_{h f}+\mathrm{C}_{h v} \mathrm{~A}_{h v} \frac{\mathrm{Q}}{2}
$$

\section{Analysis}

Equation (1) and (2) are similar to classical EOQ model in forms of expression, so is the solving method. The two objective functions are convex by Q. So, we can get the optimal solution by first order condition (FOC).

Let $\hat{Q}$ denote carbon optimization order quantity (COQ), $Q^{\times}$denote EOQ. Then,

$$
\begin{gathered}
\hat{Q}=\sqrt{\frac{2 F_{t f} A_{t f} D}{F_{h v} A_{h v}}} \\
Q^{x}=\sqrt{\frac{2 C_{t f} A_{t f} D}{C_{h v} A_{h v}}}
\end{gathered}
$$

\subsection{The order quantity in optimal solution}

From equation (3) and (4), the order quantity in optimal solution has nothing to do with fixed activities of inventory and variable activities of transport. Intuitively, fixed activities of inventory have only related with holding goods, and variable activities of transport have only related with the quantity of loading goods. The items relating the parameter in equation (1) and (2) do not include variable $Q$ in expression. So, they have nothing to do with optimal order quantity. It is the relationship between EF, cost coefficient of fixed activity of inventory and EF, cost coefficient of variable activity of transport that determines the relationship between the two optimal order quantities. So, there is theorem 1. 


\section{Theorem 1.}

(1) if $\frac{F_{t f}}{F_{h v}}>\frac{C_{t f}}{C_{h v}}$, then $\hat{Q}>Q^{x}$;

(2) if $\frac{F_{t f}}{F_{h v}}=\frac{C_{t f}}{C_{h v}}$, then $\hat{Q}=Q^{x}$;

(3) if $\frac{F_{t f}}{F_{h v}}<\frac{C_{t f}}{C_{h v}}$, then $\hat{Q}<Q^{x}$.

Proof. Comparing equation (3) and (4), we can derive Theorem 1 easily.

Theorem 1 is similar to the result of Hua et al. (2011), which is obtained in the case of carbon trade. The second item of Theorem 1 means low carbon and low cost, in which the two objectives of carbon and cost are consistent. By optimizing cost, carbon is at its optimal situation too. This ideal case is possible because carbon has positive even linear correlation with cost for many logistics activities. For example, Tian and Yang (2013) established logistics index system to measure the level of low carbon only from the perspective of energy consumption. Meanwhile, it also means that there is no chance to reduce emissions by selecting order quantity. But the other two cases are more common, which mean that there are opportunities to reduce emissions by optimizing order quantity. Then, one question appears that the one being at optimal point leads to how much the other deviates from its optimal point? That is what we will answer in the following.

\subsection{The objective value in optimal solution}

Substituting (3) and (4) into the objective function (1) and (2) respectively, we get:

$$
\begin{aligned}
& T F(\hat{Q})=F_{t v} A_{t v} D+F_{h f} A_{h f}+\sqrt{2 F_{t f} A_{t f} F_{h v} A_{h v} D} \\
& T C\left(Q^{\times}\right)=C_{t v} A_{t v} D+C_{h f} A_{h f}+\sqrt{2 C_{t f} A_{t f} C_{h v} A_{h v} D}
\end{aligned}
$$

Obviously, it is only the third item of each equation above that we can optimize by operational adjustment. So, the third item is the scope that we can influence in this research. The role of reduction by choosing order quantity depends on how much the third item occupies the objective values. Larger the proportion is, more important the role is also. 
Next, we still continue our analysis by supposing the role is important. We get the new objective function by removing the first two items. Then,

$$
\begin{gathered}
T F_{v}(\hat{\mathrm{Q}})=\mathrm{F}_{t f} \mathrm{~A}_{t f} \frac{\mathrm{D}}{\hat{\mathrm{Q}}}+\mathrm{F}_{h v} \mathrm{~A}_{h v} \frac{\hat{\mathrm{Q}}}{2} \\
T C_{v}\left(\mathrm{Q}^{\mathrm{X}}\right)=\mathrm{C}_{t f} \mathrm{~A}_{t f} \frac{\mathrm{D}}{\mathrm{Q}^{\mathrm{x}}}+\mathrm{C}_{h v} \mathrm{~A}_{h v} \frac{\mathrm{Q}^{\mathrm{x}}}{2}
\end{gathered}
$$

If order quantity deviates from optimal order quantity by $r$ times, then

$$
\begin{gathered}
T F_{v}(\mathrm{r} \hat{\mathrm{Q}})=\frac{1}{2}\left(\mathrm{r}+\frac{1}{\mathrm{r}}\right) T F_{v}(\hat{\mathrm{Q}}) \\
T C_{v}\left(r Q^{x}\right)=\frac{1}{2}\left(\mathrm{r}+\frac{1}{\mathrm{r}}\right) T C_{v}\left(\mathrm{Q}^{\mathrm{X}}\right)
\end{gathered}
$$

Obviously, if and only if $r=1$, the objective value is minimum. Otherwise it is larger. This is the robustness of classical EOQ that is well known.

$$
\mathrm{a}=\frac{\hat{\mathrm{Q}}}{\mathrm{Q}^{\mathrm{X}}}=\sqrt{\frac{\mathrm{C}_{h v}}{\mathrm{C}_{t f}} \cdot \frac{\mathrm{F}_{t f}}{\mathrm{~F}_{h v}}}
$$

For the symmetry of the problem, there is theorem 2.

Theorem 2. The relationship between cost of $C O Q$ and carbon footprint of EOQ is:

$$
\begin{aligned}
& T F_{v}\left(\mathrm{Q}^{\mathrm{X}}\right)=\frac{1}{2}\left(\mathrm{a}+\frac{1}{\mathrm{a}}\right) T F_{v}(\hat{\mathrm{Q}}) \\
& T C_{v}(\hat{\mathrm{Q}})=\frac{1}{2}\left(\mathrm{a}+\frac{1}{\mathrm{a}}\right) T C_{v}\left(\mathrm{Q}^{\mathrm{X}}\right)
\end{aligned}
$$


Proof. From equation (7) and (8), we can derive Theorem 2 easily.

Equation (10) and (11) can be shown as Figure 1. Like classical EOQ, the objective value, either carbon or cost, shows rather robustness when order quantity changes in a certain range. E.g. when $\alpha=2$, namely $\mathrm{COQ}$ is twice of EOQ, carbon emissions will be increased only by $25 \%$ if choosing EOQ, and costs will be increased only by $25 \%$ if choosing COQ.

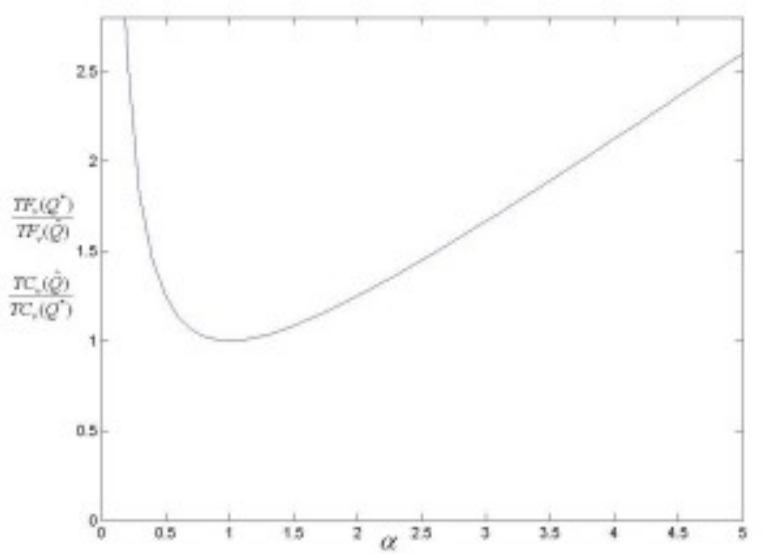

Figure 1. Ratio of objective function value and $\alpha$

According to Theorem 2, if choosing COQ, the absolute amount of costs penalty is:

$$
\Delta T C=T C_{v}(\hat{\mathrm{Q}})-T C_{v}\left(\mathrm{Q}^{\mathrm{x}}\right)=\frac{1}{2}\left(\mathrm{a}+\frac{1}{\mathrm{a}}-2\right) \sqrt{2 \mathrm{C}_{t f} \mathrm{~A}_{t f} \mathrm{C}_{h v} \mathrm{~A}_{h v} \mathrm{D}}
$$

If choosing EOQ, the absolute amount of carbon footprints penalty is:

$$
\Delta T F=T F_{v}\left(\mathrm{Q}^{\mathrm{X}}\right)-T F_{v}(\hat{\mathrm{Q}})=\frac{1}{2}\left(\mathrm{a}+\frac{1}{\mathrm{a}}-2\right) \sqrt{2 \mathrm{~F}_{t f} \mathrm{~A}_{t f} \mathrm{~F}_{h v} \mathrm{~A}_{h v} \mathrm{D}}
$$

By the two equations, it can define an economic assessment for carbon through operations adjustment, which can provide some meaningful insights for corporations in quantity. The equation is:

$$
\frac{\Delta T C}{\Delta T F}=\frac{\sqrt{\mathrm{C}_{t f} \mathrm{C}_{h v}}}{\sqrt{\mathrm{F}_{t f} \mathrm{~F}_{h v}}}
$$


Theorem 2 describes the two extreme cases of order quantity, which is equal to either COQ or EOQ. It can be extended to the general case. There are some corollaries as follows.

Corollary 1. If order quantity $Q$ is $r$ times of $C O Q$, then

$$
\begin{aligned}
& \text { (1) } T F_{v}(\mathrm{Q})=\frac{1}{2}\left(\mathrm{r}+\frac{1}{\mathrm{r}}\right) T F_{v}(\hat{\mathrm{Q}}) \\
& \text { (2) } T C_{v}(\mathrm{Q})=\frac{1}{2}\left(\mathrm{ra}+\frac{1}{\mathrm{ra}}\right) T C_{v}\left(\mathrm{Q}^{\mathrm{X}}\right)
\end{aligned}
$$

Proof. By substituting variables among equation (7), (8) and (9), we can derive Corollary 1 easily.

According to Corollary $1, r=1$ means the decision is made by optimizing carbon, where $\mathrm{Q}$ is equal to COQ, and equation of corollary $1(2)$ is equivalent to equation (11). The case of $r=1 / \alpha$ means the decision is made by optimizing cost, where $Q$ is equal to $E O Q$, and equation of corollary $1(1)$ is equivalent to equation (10).

For the symmetry of the problem, Corollary 1 can be expressed as follows.

Corollary 2. If order quantity $Q$ is $r$ times of EOQ, then

$$
\begin{aligned}
& \text { (1) } T F_{v}(\mathrm{Q})=\frac{1}{2}\left(\frac{\mathrm{r}}{\mathrm{a}}+\frac{\mathrm{a}}{\mathrm{r}}\right) T F_{v}(\hat{\mathrm{Q}}) \\
& \text { (2) } T C_{v}(\mathrm{Q})=\frac{1}{2}\left(\mathrm{r}+\frac{1}{\mathrm{r}}\right) T C_{v}\left(\mathrm{Q}^{\mathrm{X}}\right)
\end{aligned}
$$

According to Corollary 2, r=1 means $Q$ is equal to EOQ, and equation of Corollary $2(1)$ is equivalent to equation (10). The case of $r=\alpha$ means $Q$ is equal to $C O Q$, and equation of Corollary $1(2)$ is equivalent to equation (11).

According to the four equations of corollary 1 and 2, when order quantity deviates from optimal order quantity, the objective value is larger than the optimal objective value. It is as follows.

$$
\frac{T F_{v}(\mathrm{Q})}{T F_{v}(\hat{\mathrm{Q}})}>1, \frac{T C_{v}(\mathrm{Q})}{T C_{v}\left(\mathrm{Q}^{\mathrm{X}}\right)}>1
$$

This is consistent with the practical meaning of the objective function. 
Corollary 3. If order quantity $Q$ is $r$ times of $C O Q$, then

(1) If $\alpha<1$ and $r<\frac{1}{\sqrt{\alpha}}$, or if $\alpha>1$ and $r>\frac{1}{\sqrt{\alpha}}$, then $\frac{T F_{v}(\mathrm{Q})}{T F_{v}(\hat{\mathrm{Q}})}<\frac{T C_{v}(\mathrm{Q})}{T C_{v}\left(\mathrm{Q}^{\mathrm{X}}\right)}$;

(2) If $\alpha=1$ and $r=\frac{1}{\sqrt{\alpha}}$, then $\frac{T F_{v}(\mathrm{Q})}{T F_{v}(\hat{\mathrm{Q}})}=\frac{T C_{v}(\mathrm{Q})}{T C_{v}\left(\mathrm{Q}^{\mathrm{X}}\right)}$;

(3) If $\alpha<1$ and $r>\frac{1}{\sqrt{\alpha}}$, or if $\alpha>1$ and $r<\frac{1}{\sqrt{\alpha}}$, then $\frac{T F_{v}(\mathrm{Q})}{T F_{v}(\hat{\mathrm{Q}})}>\frac{T C_{v}(\mathrm{Q})}{T C_{v}\left(\mathrm{Q}^{\mathrm{X}}\right)}$.

Proof. Comparing the two equations of corollary 1, we can derive Corollary 3 easily.

Given the degree that order quantity deviates from CQO, we can judge whether the increasing of carbon footprints is larger than that of costs or not by Corollary 3. Specially, Corollary 3(1) means the effect on carbon is less than on cost, so carbon profits are larger. Corollary 3(2) means the effect on carbon is equal to on cost. Corollary 3(3) means the effect on carbon is larger than on cost, so carbon profits are smaller.

Like Corollary 1, according to equation (9), there is Corollary 4 corresponding with Corollary 3.

Corollary 4. If order quantity $Q$ is $r$ times of EOQ, then

(1) If $\alpha<1$ and $r<\sqrt{\alpha}$, or if $\alpha>1$ and $r>\sqrt{\alpha}$, then $\frac{T F_{v}(\mathrm{Q})}{T F_{v}(\hat{\mathrm{Q}})}<\frac{T C_{v}(\mathrm{Q})}{T C_{v}\left(\mathrm{Q}^{\mathrm{X}}\right)}$;

(2) If $\alpha=1$ or $r=\alpha$, then; $\frac{T F_{v}(\mathrm{Q})}{T F_{v}(\hat{\mathrm{Q}})}=\frac{T C_{v}(\mathrm{Q})}{T C_{v}\left(\mathrm{Q}^{\mathrm{X}}\right)}$

(3) If $\alpha<1$ and $r>\sqrt{\alpha}$, or if $\alpha>1$ and $r<\sqrt{\alpha}$, then $\frac{T F_{v}(\mathrm{Q})}{T F_{v}(\hat{\mathrm{Q}})}>\frac{T C_{v}(\mathrm{Q})}{T C_{v}\left(\mathrm{Q}^{\mathrm{X}}\right)}$.

The implications of Corollary 4 are similar to that of Corollary 3. 


\section{Numerical Studies}

In this section we present a series of numerical examples to illustrate the above analytical results. Due to the analysis in section 3, some parameters have nothing to do with the objective. For simplicity, we only give the data of the parameters having relevant to the objective. Let $D=60,000$, and the rest of the parameters and the results are summarized in Table 1.

\begin{tabular}{|c|c|c|c|c|c|c|c|c|c|c|c|c|c|}
\hline & $C_{t f}$ & $C_{h v}$ & $F_{t f}$ & $F_{h v}$ & $A_{t f}$ & $A_{h v}$ & $\hat{\boldsymbol{Q}}$ & $Q^{x}$ & $\alpha$ & $T F_{v}$ & $T C_{v}$ & $\frac{F_{t f}}{F_{h v}}$ & $\frac{C_{t f}}{C_{h v}}$ \\
\hline 1 & 36 & 0.3 & 60 & 0.2 & 5 & 2 & 232.38 & 146.97 & 1.58 & 92.95 & 88.18 & 300 & 120 \\
\hline 2 & 36 & 0.3 & 60 & 0.2 & 5 & 2 & 232.38 & 146.97 & 1.58 & 92.95 & 88.18 & 300 & 120 \\
\hline 3 & 40 & 0.4 & 50 & 0.2 & 5 & 2 & 212.13 & 134.16 & 1.58 & 84.85 & 107.33 & 250 & 100 \\
\hline 4 & 40 & 0.4 & 50 & 0.2 & 5 & 2 & 212.13 & 134.16 & 1.58 & 84.85 & 107.33 & 250 & 100 \\
\hline 5 & 40 & 0.2 & 60 & 0.3 & 5 & 2 & 189.74 & 189.74 & 1 & 113.84 & 75.89 & 200 & 200 \\
\hline 6 & 40 & 0.4 & 50 & 0.5 & 5 & 2 & 134.16 & 134.16 & 1 & 134.16 & 107.33 & 100 & 100 \\
\hline 7 & 50 & 0.4 & 45 & 0.5 & 5 & 2 & 127.28 & 150 & 0.85 & 127.28 & 120 & 90 & 125 \\
\hline 8 & 50 & 0.4 & 45 & 0.5 & 5 & 2 & 127.28 & 150 & 0.85 & 127.28 & 120 & 90 & 125 \\
\hline 9 & 60 & 0.4 & 50 & 0.5 & 5 & 2 & 134.16 & 164.32 & 0.82 & 134.16 & 131.45 & 100 & 150 \\
\hline 10 & 60 & 0.4 & 50 & 0.5 & 5 & 2 & 134.16 & 164.32 & 0.82 & 134.16 & 131.45 & 100 & 150 \\
\hline 11 & 60 & 0.4 & 50 & 0.5 & 5 & 2 & 134.16 & 164.32 & 0.82 & 134.16 & 131.45 & 100 & 150 \\
\hline 12 & 60 & 0.4 & 50 & 0.5 & 5 & 2 & 134.16 & 164.32 & 0.82 & 134.16 & 131.45 & 100 & 150 \\
\hline
\end{tabular}

\begin{tabular}{|c|c|c|c|c|c|c|c|c|c|}
\hline & $\operatorname{TFv}\left(Q^{x}\right)$ & $T C_{V} \hat{Q}$ & \multicolumn{2}{|c|}{$\frac{\Delta T C_{v}}{\Delta T F_{v}}$} & $\boldsymbol{r}$ & $\mathbf{T} \boldsymbol{F}_{\boldsymbol{v}}(\boldsymbol{r} * \hat{\mathbf{Q}})$ & $T C_{v}(r * \hat{Q})$ & $\mathbf{T} \boldsymbol{F}_{v}(\boldsymbol{r} * \boldsymbol{Q} *)$ & $\mathbf{T C} C_{v}(r * Q *)$ \\
\hline 1 & 4200 & 3984.47 & 0.95 & 0.95 & 1.8 & 4469.35 & 5755.35 & 3826.67 & 4240 \\
\hline 2 & 4200 & 3984.47 & 0.95 & 0.95 & 0.7 & 4038.68 & 3618.55 & 5125.71 & 3831.43 \\
\hline 3 & 3834.06 & 4849.74 & 1.26 & 1.26 & 1.8 & 4079.94 & 7005.18 & 3493.25 & 5160.76 \\
\hline 4 & 3834.06 & 4849.74 & 1.26 & 1.26 & 0.6 & 3925.98 & 4387.86 & 5221.62 & 4966.02 \\
\hline 5 & 4647.58 & 3098.39 & 0.67 & $\mathrm{NaN}$ & 1.8 & 5473.82 & 3649.21 & 5473.82 & 3649.21 \\
\hline 6 & 5477.23 & 4381.78 & 0.8 & $\mathrm{NaN}$ & 1.8 & 6450.95 & 5160.76 & 6450.95 & 5160.76 \\
\hline 7 & 5266.4 & 4965.21 & 0.94 & 0.94 & 1.8 & 6119.91 & 5344.98 & 6736.1 & 5769.91 \\
\hline 8 & 5266.4 & 4965.21 & 0.94 & 0.94 & 0.9 & 5225.02 & 5078.12 & 5205.17 & 4926.2 \\
\hline 9 & 5590.17 & 5477.23 & 0.98 & 0.98 & 2 & 6846.53 & 6024.95 & 7826.24 & 6708.2 \\
\hline 10 & 5590.17 & 5477.23 & 0.98 & 0.98 & 0.8 & 5614.16 & 5860.63 & 5478.37 & 5500.73 \\
\hline 11 & 5590.17 & 5477.23 & 0.98 & 0.98 & 0.9 & 5505.39 & 5616.62 & 5505.39 & 5394.16 \\
\hline 12 & 5590.17 & 5477.23 & 0.98 & 0.98 & 1.11 & 5505.39 & 5394.16 & 5732.44 & 5394.16 \\
\hline
\end{tabular}

Table 1. Results of the examples

The results in Table 1 verify Theorem 1-2 and Corollary 1-4. They show that the relationship between EOQ $\left(Q^{\times}\right)$and COQ $(\hat{Q})$ was determined by the relationship between $\frac{F_{t f}}{F_{h v}}$ and $\frac{C_{t f}}{C_{h v}}$. In 
the case of $\frac{F_{t f}}{F_{h v}} \neq \frac{C_{t f}}{C_{h v}}$, we get $\mathrm{Q}^{\times} \neq \hat{Q}$ which means there are opportunities to reduce emissions by choosing order quantity. In the case of $\frac{F_{t f}}{F_{h v}}=\frac{C_{t f}}{C_{h v}}$, the objective of cost is consistent with the objective of carbon, and there are no opportunities to reduce emissions by operations adjustment.

If we obtain the order quantity only by one objective, such as carbon or cost, it will lead to the value of the other objective increasing. But the increasing ratio, which only depends on $\alpha$, is symmetrical for each of the two objectives. In Table 1 , there are also the increasing quantities of each objective, which can act as the economic assessment standard for carbon emission. The data in Table 1 shows that the value of economic assessment standard of carbon emission does not exist when $\alpha=1$, because it has already been at its optimal point for each objective.

In general, we don't choose the order quantity by one objective only, but by the trade-off between the two objectives. In Table 1, we calculate the ratio of one objective to the other. The results verify Corollary $1-4$. When we choose order quantity $r$ times of COQ, in the case of $\alpha>1$ or $\alpha<1$, there exists a certain range of $r$. If $r$ is within the range, the change or increasing of carbon emissions is less than that of costs. Otherwise, the increasing of carbon emissions is larger. Only in the case of $\alpha=1$ or $r^{2}=1$, the changes of carbon emissions is equal to that of costs. When choosing order quantity $r$ times of EOQ, we can get the similar results from the data in Table 1. All these can help logistics organizations to make trade-off between costs and carbon emissions in quantity.

\section{Conclusions}

There have emerged a large number of literatures about low carbon logistics, most of which take carbon footprint as an additional source of costs. The general method is to estimate or assume the economic coefficients of carbon footprint and incorporate it into the economic objective function, and then to analyze the model in the traditional logistics theorem framework. In addition, most researches are for the policies of carbon trade and carbon cap. From the literatures reviewed, we find that the carbon market is very small still and some researches have proved that carbon market cannot be effective for reducing emissions unless carbon price is extremely high. Meanwhile, the policy of carbon cap has not been put into practice in most countries. So the two settings, on which most researches have done, are very different from the current social status. However, the paper also indicates optimistically that even if the traditional factors, such as economic trade-off and the pressure from government regulations, are not valid, the pressure and motivation of reduction for corporations are still growing, which are mainly from corporation social responsibility and increasing awareness of 
low carbon among consumers and society. So unlike the traditional research, we put our research in the background of corporation being social responsibility and consumers or society pursuing low carbon, and then take carbon footprint as objective directly to search out the reduction opportunities by choosing order quantity. Though we assume that corporation has the motivation to reduce emissions voluntary, economic factor is still necessary for it to survive, develop and achieve its visions. So, we also take the research on EOQ as a reference. By the comparative analysis study and a series of numerical examples, we obtained some meaningful insights. The optimal order quantity, either EOQ or COQ, has nothing to do with fixed activities of inventory and variable activities of transport. Whether EOQ is larger than $\mathrm{COQ}$ or not depends on the relative values of $\mathrm{EF}$, cost coefficient of fixed activity of inventory and $E F$, cost coefficient of variable activity of transport. In extreme cases, which order quantity is equal to EOQ or COQ, the ratio of total costs and carbon footprints is rather robust. We also obtain some corollaries in general case when order quantity is not equal to EOQ or COQ. If order quantity deviates from EOQ or $\mathrm{COQ}$ by $r$ times, these corollaries can be employed to judge whether the increasing ratio to the optimal value of cost is larger than that of carbon or not, which can be as the foundation for corporations to make trade-offs between cost and carbon. All these can help the corporations with social responsibility and wish of voluntary reduction to make rational decision. There are some topics for further research in this area. In this paper, we assumed that the demand is deterministic and the lead time is zero. The further research can be extended to take into account more factors influencing inventory or transport considered in the classical EOQ framework, such as stochastic demand, constant or variable lead time, discounts, and etc.

\section{Acknowledgments}

This work is supported by Beijing Natural Science Foundation (9133018) and Beijing Key Laboratory of Intelligent Logistics System, Beijing Wuzi University. 


\section{References}

Arslan, M.C., \& Turkay, M. (2010). EOQ Revisited with Sustainability Considerations. Working paper.

Battini, D., Persona, A., \& Sgarbossa, F. (2013). A Sustainable EOQ Model: theoretical formulation and applications. International Journal of Production Economics, available online 15 August 2013.

BearingPoint, 4th Supply Chain Monitor, 2010-2011, available online at http://www.bearingpointconsulting.com/de-de/download/TAP-SC EN.pdf (date of last access October 6th, 2013).

Benjaafar, S., Yanzhi, L., \& Daskin, M. (2013). Carbon Footprint and the Management of Supply Chains: Insights From Simple Models. IEEE Transactions on Automation Science and Engineering, 10(1), 99-116. http://dx.doi.org/10.1109/TASE.2012.2203304

Blengini, G.A., \& Shields, D.J. (2010). Green Labels and Sustainability Reporting. Management of Environmental Quality: An International Journal, 21(4), 477-493.

http://dx.doi.org/10.1108/14777831011049115

Bouchery, Y., Ghaffari, A., Jemai, Z., et al. (2012). Including sustainability criteria into inventory models. European Journal of Operational Research, 222(2), 229-240. http://dx.doi.org/10.1016/j.ejor.2012.05.004

Cachon, G.P. (2013). Retail store density and the cost of greenhouse gas emissions. Working paper.

Carlsson, J.G., \& Jia, F. (2012). Minimizing emissions in facility location. University of Minnesota working paper.

Caro, F., Charles, J.C., \& Tan, T., \& Rob, Z. (2011). Carbon-Option and Carbon-Neural Supply Chains. Working paper. http://dx.doi.org/10.1596/1813-9450-5878

Chen, X., Benjaafar, S., \& Elomri, A. (2012). The carbon-constrained EOQ. Operations Research Letters, 41(2), 172-179. http://dx.doi.org/10.1016/j.orl.2012.12.003

Daganzo (2010). Logistics systems analysis. Beijing. Publishing house of electronics industry.

Hoen, K.M.R., Tan, T., Fransoo, J.C., et al. (2011). Effect of carbon emission regulations on transport mode selection in supply chains. Eindhoven University of Technology.

Hua, G., Cheng, T.C.E., \& Wang, S. (2011). Managing carbon footprints in inventory management. International Journal of Production Economics, 132(2), 178-185. http://dx.doi.org/10.1016/j.ijpe.2011.03.024 
Lieb, K.J., \& Lieb, R.C. (2010). Environmental sustainability in the third-party logistics (3PL) industry. International Journal of Physical Distribution \& Logistics Management, 40(7), 524-533. http://dx.doi.org/10.1108/09600031011071984

Tian, Y., \& Yang, M. (2013). Demonstration analysis for the low-carbon factors index system of logistics enterprises. Journal of Industrial Engineering and Management, 6(1), 297-307. http://dx.doi.org/10.3926/jiem.681

Wakeland, W., Sears, L., \& Venkat, K. (2009). Measuring the Effects of Carbon Footprint Training on Consumers. Sustainability: The Journal of Record, 2(1), 45-52. http://dx.doi.org/10.1089/SUS.2009.9900

Journal of Industrial Engineering and Management, 2014 (www.jiem.org)

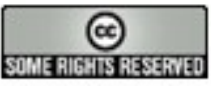

Article's contents are provided on a Attribution-Non Commercial 3.0 Creative commons license. Readers are allowed to copy, distribute and communicate article's contents, provided the author's and Journal of Industrial Engineering and Management's names are included. It must not be used for commercial purposes. To see the complete license contents, please visit http://creativecommons.org/licenses/by-nc/3.0/. 